\title{
Congenital long QT syndrome of particularly malignant course connected with so far unknown mutation in the sodium channel SCN5A gene
}

\author{
Beata Uziębło-Życzkowska ${ }^{1}$, Dariusz Michałkiewicz ${ }^{1}$, \\ Anna Jackun-Podleśna ${ }^{1}$, Grzegorz Gielerak ${ }^{1}$, Agnieszka Zienciuk-Krajka² \\ ${ }^{1}$ Department of Cardiology and Internal Diseases, Military Institute of Medicine, Warsaw, Poland \\ ${ }^{2}$ Department of Cardiology and Electrotherapy, Medical University of Gdansk, Poland
}

\begin{abstract}
This article presents the case of a 35 year-old male with long QT syndrome (LQTS) who suffered from sudden cardiac arrest. Even though asymptomatic LQTS had been diagnosed, the patient had not undergone any medical treatment. His two daughters, aged four and seven, were also diagnosed with LQTS. A new, previously unknown, mutation of the SCN5A gene has been found in the family. The older daughter died suddenly before implantable cardioverterdefibrillator (ICD) implantation, but the father and the younger daughter have been implanted with ICDs. (Cardiol J 2013; 20, 1: 78-82)

Key words: long QT syndrome, sudden cardiac arrest, new mutation gene SCN5A in LQTS
\end{abstract}

\section{Introduction}

The long QT syndrome (LQTS), apart from QT interval > $440 \mathrm{~ms}$ at basal ECG, is characterized by a higher risk of polymorphic ventricular tachycardia of torsade de pointes type and sudden cardiac death (SCD). The cause of LQTS is abnormalities of cardiac ion channels (sodium and potassium) determined genetically or acquired in another way (for example by drugs). The diagnosis of LQTS is made on the basis of ECG findings and family interview. The first manifestation of congenital LQTS can occur as a syncope or sudden cardiac arrest (SCA).

This article presents the case of a patient who had suffered from LQTS and experienced SCA. In the case of one of the patient's daughters, SCD occurred. It is noteworthy that an unknown mutation of SCN5A gene was found in the family.

\section{Case report}

The patient is a 35 year-old previously healthy male who was admitted to hospital after a SCA episode during ventricular fibrillation (VF). His past medical history was unremarkable. The SCA caused by VF occurred during a soccer match. The patient was successfully reanimated by an emergency medical team with several successive defibrillation shocks. In the following days of hospitalization, a prolonged QT interval up to $566 \mathrm{~ms}$ at basal ECG was observed (Fig. 1). The dose of nonselective beta-blocker (propranolol), administered to the patient, was increased up to the maximum. Despite treatment, the patient suffered from multiple arrhythmia recurrences - ventricular tachycardia often degenerating into VF. Therefore, right ventricular pacing was used at a frequency of $100 / \mathrm{min}$.

Address for correspondence: Beata Uziębło-Życzkowska, MD, PhD, Department of Cardiology and Internal Diseases, Military Institute of Medicine, ul. Szaserów 128, 04-141 Warszawa, Poland, e-mail: buzieblo-zyczkowska@wim.mil.pl 


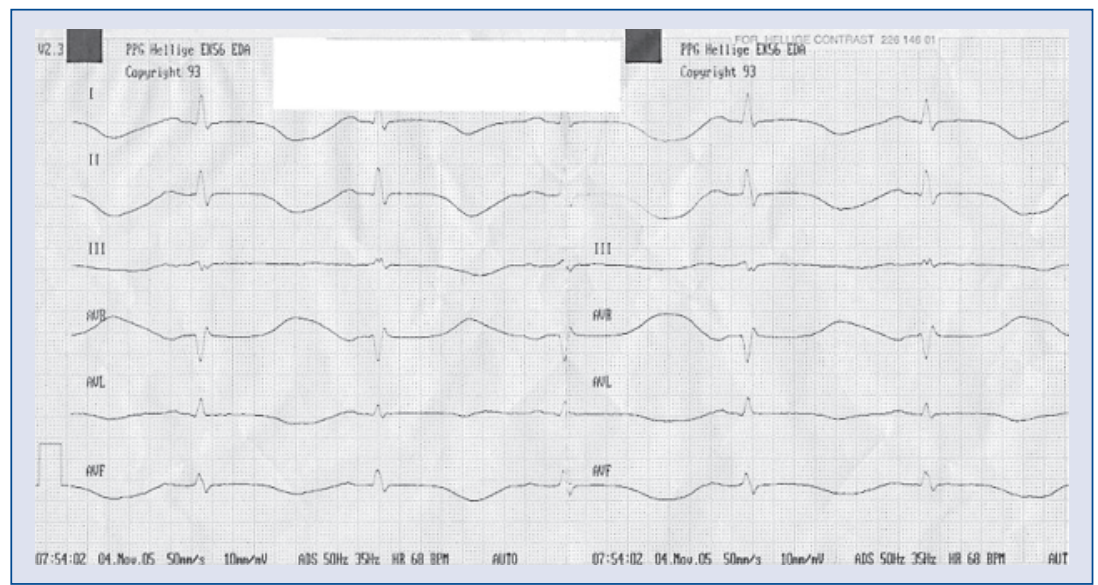

Figure 1. Resting ECG registration on the first day of hospitalization; $50 \mathrm{~mm} / \mathrm{s}$; major QTc prolongation (QTc calculated by Bazett's formula $=566 \mathrm{~ms}$ ).

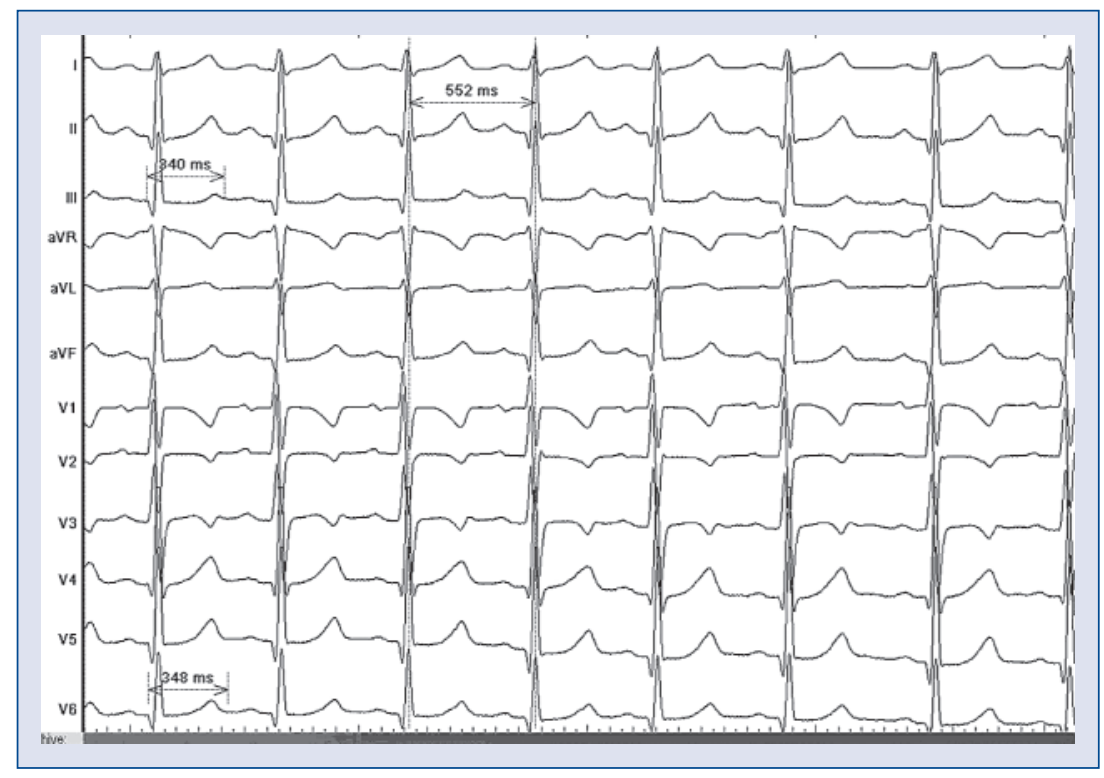

Figure 2. The four year-old daughter's ECG registration; $50 \mathrm{~mm} / \mathrm{s}$; QTc interval = $457 \mathrm{~ms}$.

Simultaneously, a decrease in beta-blocker dose was implemented. No arrhythmia recurrences were observed by the tenth day. The patient was implanted with a cardioverter-defibrillator (ICD) with the function of DDD stimulation. Initially, the stimulation was $100 / \mathrm{min}$, but over the following days this was slowly decreased.

Having interviewed the patient's relatives, it turned out that prolongation of QT interval had been observed at the patient's previous basal ECG. At that time, no treatment had been implemented. The patient was an active sportsman with no history of syncopes. The patient's younger daughter aged four was noticed to have a borderline QT interval (Fig. 2) with no disease symptoms, whereas his older daughter aged seven was diagnosed with LQTS (Fig. 3) and beta-blocker treatment was implemented. She had fainted only once prior to the diagnosis of LQTS.

Molecular testing of KCNQ1, KCNH2, SCN5A, KCNE1 and KCNE2 genes was performed, and K1477N mutation in the SCN5A gene was found in the described patient and his two children. This gene variant had not been previously described as a mutation causing the long type 3 QT syndrome. The lack of data in the literature and the lack of a population control for this part of the SCN5A gene made it impossible to state clearly whether the LQTS syndrome was caused by the said change. 


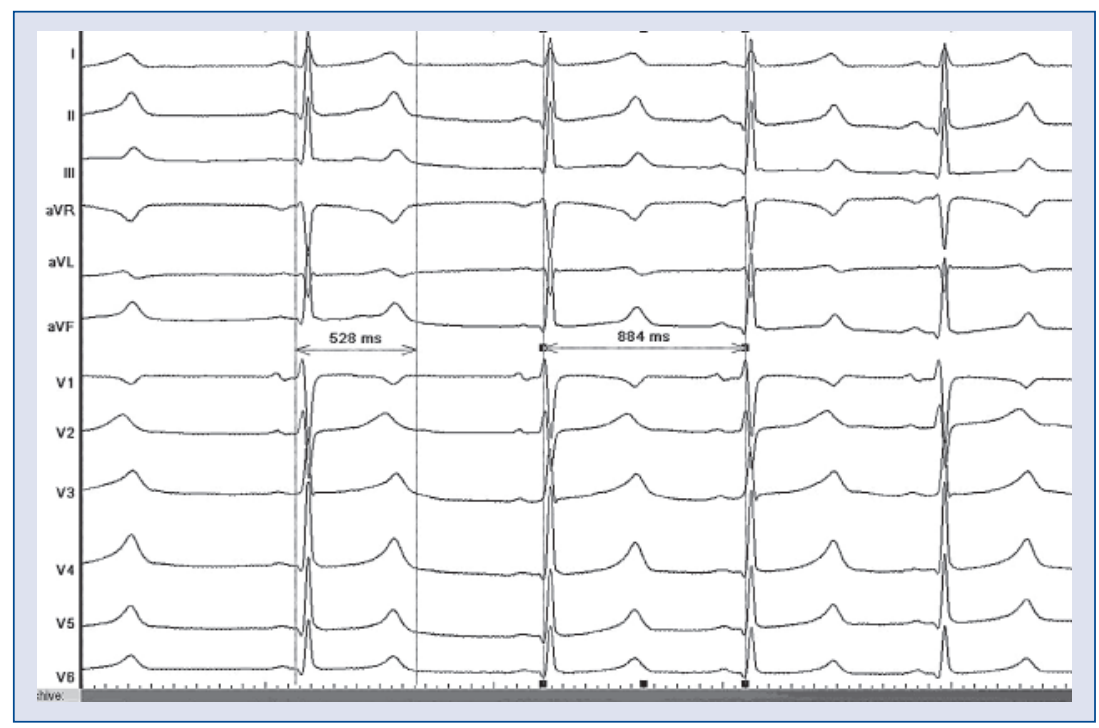

Figure 3. The seven year-old daughter's ECG registration; $50 \mathrm{~mm} / \mathrm{s}$; QTc interval = $561 \mathrm{~ms}$.

Taking into consideration the episode of SCA in the described patient, his two daughters were advised to report to the Tertiary Referral Pediatric Hospital immediately for genetic examination. Several months after obtaining the results of the genetic examination, the older patient's daughter suddenly died at home. The younger one was implanted with an ICD in the following months.

During the routine follow up (three years) there has been no cardioverter discharge and in the cardioverter memory no ventricular arrhythmias have been noted.

\section{Discussion}

The diagnostic criteria of LQTS are known in the form of a point scale, which includes electrocardiographic findings, clinical symptoms, and family history [1]. However, it is well-known that the sensitivity of such criteria is very low, so genetic testing is considered to be the most sensitive method of diagnosing LQTS [2].

There are some correlations between genotype and clinical manifestation of LQTS [3]. The differences relate to the age at which the first manifestation of the disease occurs, trigger factors, and the method of treatment. It is worth noting that the LQT3 type is associated with mutations in the sodium channel gene. Unlike LQT1 and LQT2, LQT3 can lead to life-threatening ventricular arrhythmias, mainly during sleep or at rest. Moreover, it is characterized as being of poor prognosis and limited efficacy of beta-blockers therapy [3]. A higher efficacy in treating LQT3 is obtained by combining beta-blockers with mexiletine. However, such treatment is not equally effective in all patients [4]. The manifestations described above are typical clinical manifestations of LQT3. Nevertheless, in some cases, the symptoms observed show features that are typical to two different types of LQTS. In our patient, SCA occurred while doing exercise, something that is characteristic of LQT1. On the other hand, the administration of high doses of beta-blocker showed no response. It can even be stated that the increase of the dose caused more frequent polymorphic ventricular tachycardias. Right ventricular pacing at a high frequency $(100 / \mathrm{min})$ resulted in regression of arrhythmia recurrences. This is a characteristic image of LQT3, where the frequency of arrhythmia relapses treated by beta-blockers reaches $50 \%$ [3].

Despite still developing molecular diagnostics, it should be noted that molecular testing has shown a low penetration in LQTS (around 25\%) [5], which frequently results in an asymptomatic course of the disease. Moreover, the fact that many genetic changes responsible for the incidence of LQTS remain unrecognized makes the diagnosis even more difficult.

In the described case report, molecular testing showed the presence of a new genetic change in the SCN5A gene not related to LQTS so far. Having taken into account the particularly malignant course of the LQTS (SCA in the father's case and SCD in the daughter's case), further research aimed at explaining the pathogenic role of the described mutation is of vital importance. 
Regarding genetic testing, patient genomic DNA was analyzed for mutations in all translated exons, including splice sites and adjacent regions, of the five major LQTS-susceptibility genes using a polymerase chain reaction and denaturing highperformance liquid chromatography, followed by DNA sequencing. As a result, K1477N mutation in the SCN5A gene was found in the patient and his two children. No other presumed mutation or high risk single nucleotide polymorphisms have been found in the proband. It must be noted, however, that nearly $2 \%$ of healthy Caucasians and $5 \%$ of healthy non-Caucasians host rare missense SCN5A variants, which makes the proper interpretation of genetic test results a real challenge [6].

Heterologous expression studies of ion channel variants allow the in vitro assessment as to whether the mutation confers a perturbed phenotype. However, due to methodological constraints, the method is not suitable for regular clinical testing, and to date less than $20 \%$ of all published LQTS-causing mutations have been characterized functionally $[7,8]$.

In our case, in vitro evaluation of $\mathrm{K} 1477 \mathrm{~N}$ has not been done since this research tool was not available to the authors. On the other hand, the number of family members available for testing was too low to perform a co-segregation study.

It is clear that knowledge about the specific gene-encoded regions is a vital determinant of the likelihood of pathogenicity. In our case, deliberations on a possible influence of $\mathrm{K} 1477 \mathrm{~N}$ variant on SCN5A-encoded NaV1.5 sodium channel must be based on the location of the mutation within the gene, and a review of all published data on SCN5A-gene variants. The $\mathrm{K} 1477 \mathrm{~N}$ mutation is located in an interdomain linker (IDL) between domains DIII and DIV, which is known to be important for current inactivation and to be involved in determining a LQT3 phenotype [7]. In the paper by Kapa et al. [8], of 28 SCN5A mutations, only four were found in IDL DIII/DIV, one of them being a rare control.

On the other hand, in an international compendium of mutations in the SCN5A gene in patients referred for Brugada syndrome genetic testing, only six mutations were placed in IDL DIII/DIV [9]. Interestingly, Grant et al. [10] described a family with sudden death and features resembling LQTS, Brugada syndrome, and conduction system disease at the same time. In this family, K1500del, also located in the intracellular linker DIII-DIV, accounted for the complex phenotype. To the best of our knowledge, the K1477N mutation was absent from all previously published reports and all published databases listing the SCN5A channel common polymorphisms.

In order to treat patients suffering from LQTS, beta-blockers are firstly used. Nevertheless, as stated above, their efficacy in LQT3 is questionable. Despite beta-blocker therapy, the risk of death among patients from the high-risk group (understood as patients after SCA or syncope episodes) is up to $14 \%$ within five years [11]. Therefore, in the case of such patients, the only way of minimizing SCA is the implantation of a ICD [12].

The evaluation of SCA in asymptomatic patients still remains a challenge. The indication to implant an ICD within primary prevention is the controversial subject of much discussion. The following are considered to be the main SCD factors in LQT3 [13]: returning syncopes, beta-blockers intolerance, and family history of sudden death or LQTS. Young age is also considered to be a factor causing the risk of dangerous arrhythmias in LQTS [14]. One of the most difficult decisions is the implantation of an ICD in children. The long future lifespan and hence the large number of procedures of ICD and electrode replacement are a great burden on the patient. Furthermore, about $20-30 \%$ of ICD discharges are inappropriate. This can influence young people's minds and can lead to a dramatic deterioration of life quality, and ultimately to suicide [15]. On the other hand, taking into account the serious consequences of delaying the decision of invasive therapy (the death of the patient's daughter prior to the final decision as to ICD implantation) this method should be seriously considered and used in the treatment of both adults and children.

\section{Conclusions}

In our case, we observed the congenital form of LQTS caused by a previously unknown SCN5A gene mutation. This case report confirms that this genetic mutation might be life-threatening. Thus, our findings accord with those of other authors who have reported that the presence of a mutation in the SCN5A gene requires the implantation of an ICD, even in asymptomatic patients [16].

Taking into account that the interdomain linker DIII-DIV may have different biophysical properties than those of other IDLs, such mutations are more likely to be deleterious. The higher certainty of disease, the more likely it is that a mutation is disease-causing. We therefore suppose that K1477N could be considered a possible LQTS-causative mutation. Certainly, independent validations of 
pathogenicity with in vitro functional analysis are needed before this can be upgraded to the list of highly probable deleterious mutations.

\section{Conflict of interest: none declared}

\section{References}

1. Schwartz PJ, Moss AJ, Vincent GM, Crampton RS. Diagnostic criteria for the long QT syndrome. An update. Circulation, 1993; 88: 782-784.

2. Napolitano C, Priori SG, Schwartz PJ et al. Genetic testing in the long QT syndrome: Development and validation of an efficient approach to genotyping in clinical practice. JAMA, 2005; 294: 2975-2980.

3. Schwartz PJ, Priori SG, Spazzolini C et al. Genotype-phenotype correlation in the long-QT syndrome: Gene specific triggers for life-threatening arrhythmias. Circulation, 2001; 103: 89-95.

4. Schwartz PJ. The congenital long QT syndromes from genotype to phenotype: Clinical implications. J Intern Med, 2006; 259: 39-47.

5. Priori SG, Napolitano C, Schwartz PJ. Low penetrance in the long-QT syndrome: Clinical impact. Circulation, 1999; 99: 529-533.

6. Ackerman MJ, Splawski I, Makielski JC et al. Spectrum and prevalence of cardiac sodium channel variants among black, white, Asian, and Hispanic individuals: Implications for arrhythmogenic susceptibility and Brugada/long QT syndrome genetic testing. Heart Rhythm, 2004; 1: 600-660.

7. http://www.fsm.it/cardmoc.
8. Kapa S, Tester DJ, Salisbury BA et al. Genetic testing for long QT syndrome: Distinguishing pathogenic mutations from benign variants. Circulation, 2009; 120: 1752-1760.

9. Kapplinger JD, Tester DJ, Alders $\mathrm{M}$ et al. An international compendium of mutations in the SCN5A-encoded cardiac sodium channel in patients referred for Brugada syndrome genetic testing. Heart Rhythm, 2010; 7: 33-46.

10. Grant AO, Carboni MP, Neplioueva V et al. Long QT syndrome, Brugada syndrome, and conduction system disease are linked to a single sodium channel mutation. J Clin Invest, 2002; 110: 1201 -1209 .

11. Moss AJ, Zareba W, Hall J et al. Effectiveness and limitations of beta-blocker therapy in congenital long-QT syndrome. Circulation, 2000; 101: 616-613.

12. Groh WJ, Silka MJ, Oliver RP, Halperin BD, McAnulty JH, Kron J. Use of implantable cardioverter-defibrillators in the congenital long QT syndrome. Am J Cardiol, 1996; 78: 703-706.

13. Zipes DP, Camm AJ, Borggrefe M et al. ACC/AHA/ESC 2006 guidelines for management of patients with ventricular arrhythmias and the prevention of sudden cardiac death: Executive summary. Eur Heart J, 2006; 27: 2099-2140.

14. Hobbs JB, Peterson DR, Moss AJ et al. Risk of aborted cardiac arrest or sudden cardiac death during adolescence in the long-QT syndrome. JAMA, 2006; 296: 1249-1254.

15. Wolf MJ, Zeltser IJ, Salerno J et al. Electrical storm in children with an implantable cardioverter defibrillator: Clinical features and outcome. Heart Rhythm, 2007; 4: S43.

16. Etheridge SP, Sanatani S, Cohen MI, Albaro CA, Saarel EV, Bradley DJ. Long QT syndrome in children in the era of implantable defibrillators. J Am Coll Cardiol, 2007; 50: 1335-1340 . 